\title{
3D modeling of stellar atmospheres and the impact on the understanding of the reliability of elemental abundances in stars as tracers of galactic chemical evolution
}

\author{
H.-G. Ludwig ${ }^{1,3}$, M. Steffen ${ }^{2}$, P. Bonifacio ${ }^{3}$, E. Caffau ${ }^{1,3}$, \\ A. Kučinskas ${ }^{4}$, and B. Freytag ${ }^{5}$ \\ ${ }^{1}$ Zentrum für Astronomie der Universität Heidelberg, Landessternwarte, Königstuhl 12, \\ D-69117 Heidelberg, Germany \\ ${ }^{2}$ Leibniz Institut für Astrophysik Potsdam, An der Sternwarte 16, D-14482 Potsdam, Germany \\ ${ }^{3}$ GEPI Observatoire de Paris, CNRS, Université Paris Diderot, F-92195 Meudon Cedex, France \\ ${ }^{4}$ Vilnius University Institute of Theoretical Physics and Astronomy, A. Goštauto 12, Vilnius \\ LT-01108, Lithuania \\ ${ }^{5}$ Centre de Recherche Astrophysique de Lyon, UMR 5574, CNRS, Université de Lyon, École \\ Normale Supérieure de Lyon, 46 allée d'Italie, F-69364 Lyon Cedex 07, France
}

\begin{abstract}
We present a critical review of the construction of 3D model atmospheres with emphasis on modeling challenges. We discuss the basic physical processes which give rise to the effects which set 3D models apart from 1D standard models. We consider elemental abundances derived from molecular features, and the determination of the microturbulence with 3D models. The examples serve as illustration of the limitations inherent to 1D, however, also to 3D modeling. We find that 3D models can provide constraints on the microturbulence parameter, and predict substantial corrections for abundances derived from molecular species.
\end{abstract}

Keywords. stars: late-type, stars: atmospheres, stars: abundances, radiative transfer, convection, hydrodynamics, line: formation

\section{Introduction}

Three-dimensional radiation-hydrodynamical model atmospheres (henceforth "3D models" for short) were developed and are continuously improved to put the interpretation of observed spectra of cool, late-type stars onto a firmer footing. In comparison to standard (one-dimensional) model atmospheres the necessary compute time is much greater making the construction of models more cumbersome. However, nowadays the available computing resources together with algorithmic progress make the construction of 3D model grids possible. This, in turn, allows to apply synthetic spectra based on 3D models to the interpretation of the majority of spectra of late-type stars. This has some impact on the analysis of data taken in large spectroscopic surveys like the LAMOST survey or the Gaia-ESO survey.

In this paper we give a brief account of 3D models, and want to discuss two issues that make 3D models particularly pertinent to survey work where typically spectra of modest spectral resolution and limited signal-to-noise ratio $(\mathrm{S} / \mathrm{N})$ are acquired: the determination of the microturbulence parameter, and the determination of abundances from molecular lines. This leaves out aspects that raised some attention in recent years. Here, we have in mind the establishment of the solar abundance benchmark (see Asplund et al. 2004, 2009; Caffau et al. 2008, 2011a), and the presence - or rather absence - of the light 


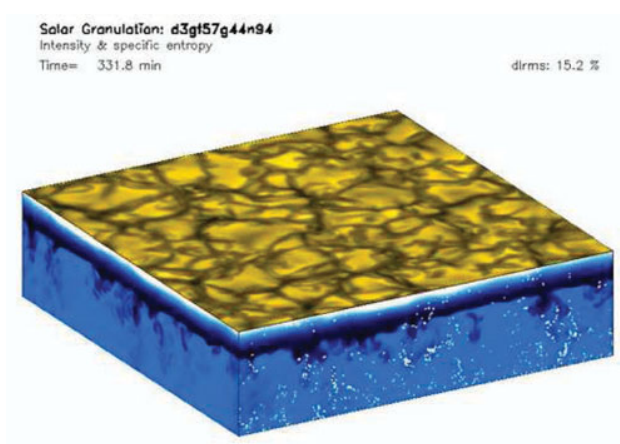

Figure 1. False-color representation of the thermodynamic properties of a snapshot taken from a solar 3D model run. The entropy is shown in blue-black tones, and the emergent intensity in yellow-black tones. White dots are tracer particles.

isotope of lithium $\left({ }^{6} \mathrm{Li}\right)$ in metal-poor halo stars (see Asplund et al. 2006; Cayrel et al. 2007; Steffen et al. 2012; Lind et al. 2013). While interesting results in their own right they were derived from non-survey grade spectra of high-resolution and high $\mathrm{S} / \mathrm{N}$.

\section{1D and 3D model atmospheres of late-type stars}

Late-type stars possess outer convective envelopes which hydrodynamical flows in the their atmospheres. This feature motivated the development of $3 \mathrm{D}$ models that can account for gas-dynamical effects which are neglected - or treated in a parameterized way - in 1D models. Figure 1 shows a typical snapshots obtained during the evolution of a solar 3D model. Table 1 summarizes the different physical assumptions going into the construction of $1 \mathrm{D}$ and $3 \mathrm{D}$ model atmospheres. The table leaves out the many technical details which need to be considered in model coding, and are in fact a topic in their own right. However, we want to emphasize three points which are of relevance later: 1) The frequency-dependence of the radiative energy transport is treated in great detail in $1 \mathrm{D}$ models. The more complex geometry of 3D models makes the solution of the transfer equation computationally more demanding so that one is forced to save time by reducing the frequency resolution. Comparing the numbers given in the Tab. 1 it is evident that the severe reduction of frequency has some impact on the final accuracy of 3D models. 2) The statistically quasi-stationary state that the 3D models are developing into is accompanied by substantial spatial and temporal fluctuations - a feature which is completely absent in 1D models. Fluctuations are a recurrent theme in the further discussion. 3) The CPU time for calculating a model is higher by several orders of magnitude in $3 \mathrm{D}$. This is the reasons why only rather recently $3 \mathrm{D}$ model atmosphere grids came into existence, and - as we shall see - are still much coarser than standard 1D grids. Nevertheless, they are now allowing the interpretation of most late-type spectra taken in extensive spectroscopic surveys.

\subsection{Abundance corrections}

One of the most important data products are 3D abundance corrections, i.e., differences that can be applied to $1 \mathrm{D}$ abundance results to correct for $3 \mathrm{D}$ effects. They are commonly derived by comparing $1 \mathrm{D}$ and $3 \mathrm{D}$ models of the same atmospheric parameters, effective temperature, surface gravity, and overall metallicity. To fully characterize the correction further, parameters entering the 1D calculations have to be specified: the mixing-length parameter entering the $1 \mathrm{D}$ models as such, and the microturbulence for spectroscopic 
Table 1. Model methodology. CT stands for convective, RT for radiative energy transport.

\begin{tabular}{ll}
\hline 1D model atmospheres & 3D model atmospheres \\
\hline plane-parallel or spherical symmetry & $\begin{array}{l}\text { no assumptions on symmetry } \\
\text { small representative patch of surface layers } \\
\text { time-dependent }\end{array}$ \\
time-independent & solution of M(HD) equations \\
CT with mixing-length theory & up-to 25 frequency bands (or bins) \\
RT with up-to $10^{5}$ frequencies & follows flow evolution \\
radiative-convective equilibrium & quasi-stationary, spatial and temporal fluctuations \\
& $10^{5} \ldots 10^{6}$ min
\end{tabular}

synthesis calculations in $1 \mathrm{D}$. The correction is calculated for each line in question by performing a synthesis of spectral line in $1 \mathrm{D}$ and $3 \mathrm{D}$ - in $3 \mathrm{D}$ demanding the averaging over many individual spectra calculated along various lines-of-sight and instances in time. The 3D-1D abundance correction is the difference of the abundance in 3D and $1 \mathrm{D}$ for a given line strength (equivalent width).

For further diagnostics often a $\langle 3 \mathrm{D}\rangle$ model is considered which is obtained by horizontal and temporal averaging of the full 3D data blocks. Like a $1 \mathrm{D}$ model it is a one-dimensional profile. The velocity field is usually replaced by a microturbulence. $3 \mathrm{D}-\langle 3 \mathrm{D}\rangle$ corrections between $3 \mathrm{D}$ and $\langle 3 \mathrm{D}\rangle$ models quantify the effects due to horizontal inhomogeneities only. Similarly, $\langle 3 \mathrm{D}\rangle-1 \mathrm{D}$ corrections allow to single out the effects stemming from differences in the mean profiles.

\subsection{Convective overshooting and its consequences}

The perhaps most important prediction of the hydrodynamical modelling of late-type atmospheres is the ubiquity of strong overshoot from the convective envelope into the optically thin photospheric layers which are stable against convection according to the Schwarzschild criterion. Indeed, 1D models follow this reasoning and assume that no macroscopic gas motions take place in the Schwarzschild-stable regions leaving radiation as the sole energy transport mechanism there. The left frame of Fig. 2 illustrates this point for two F-dwarf models $\left(T_{\text {eff }}=6500 \mathrm{~K}, \log g=4.5\right)$ of different metallicity. In the case of the $1 \mathrm{D}$ models the convective velocities as given by mixing-length theory are confined to the deep layers. In the 3D models for which averages of the vertical velocity component are depicted a pronounced penetration of the velocity field into the optically thin layers is evident. This overshooting is not very much dependent on metallicity. The metal-poor as well as solar-metallicity model exhibit a similar behavior. The overshooting velocities necessitate the introduction of macro- and microturbulence in spectral analyses in 1D.

While the amplitude of the overshooting velocity field is not very metallicity-dependent the response of the atmospheric temperature is (see Fig. 2, right panel). In 1D, the temperature of the Schwarzschild-stable layers is governed by the assumption of radiative equilibrium. In $3 \mathrm{D}$, it is controlled by a competition between cooling by overshooting motions and heating by radiation; radiation tries to establish radiative equilibrium conditions while convection works towards an adiabatic stratification. Dependent on the atmospheric parameters, in particular metallicity, the coupling between radiation and matter as given by the opacity is quite different. At low metallicity the lack of spectral lines make the coupling quite loose and the radiative heating process inefficient. At low metallicity the higher photospheric layers are cooled with respect to radiative equilibrium conditions. At solar metallicity the temperature is more tightly coupled to radiative equilibrium, and the cooling is much less pronounced. We emphasize that the reasoning 

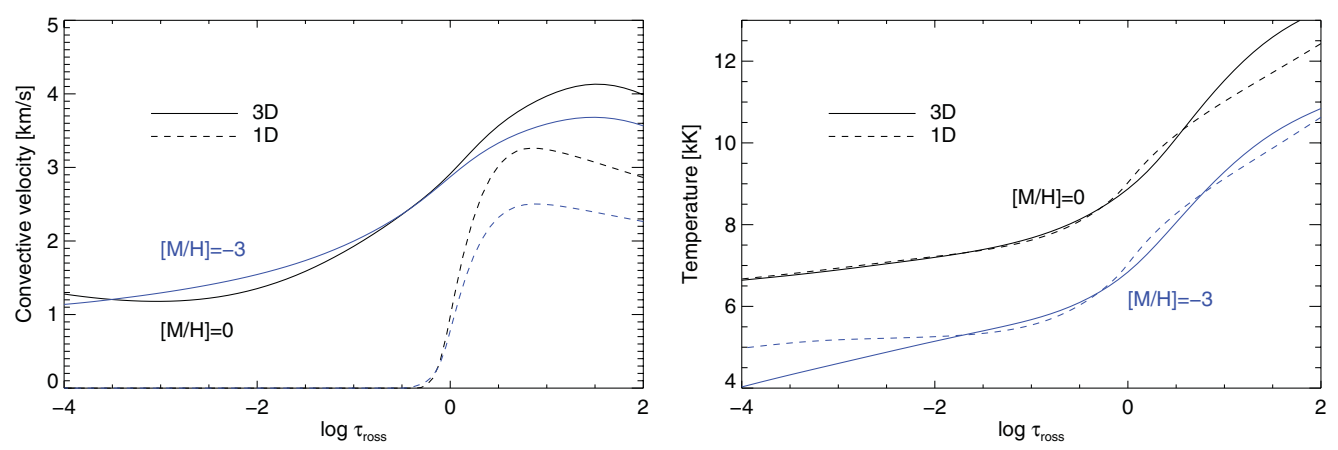

Figure 2. Comparison of velocity (left panel) and temperature (right panel) profiles as function of optical depth in two F-dwarf model atmospheres. For details see text.
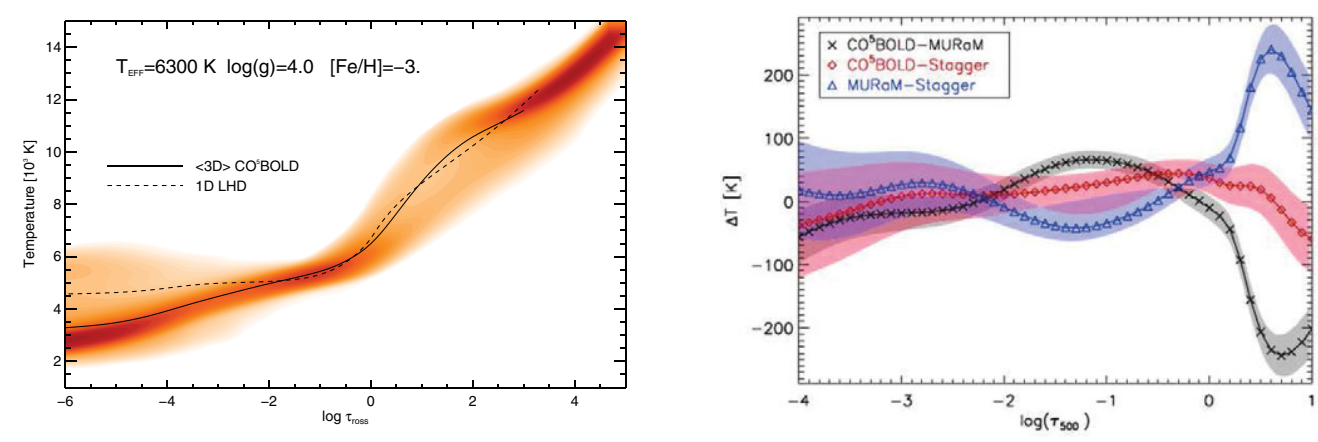

Figure 3. Left panel: Temperature profiles and 3D temperature fluctuations (shades of red) in a metal-poor F-dwarf 3D model atmosphere (from González Hernández et al. 2010; (C)ESO, reproduced with permission). Right panel: Comparison of temperature profiles of solar 3D models calculated with different codes (from Beeck et al. 2012; (c)ESO, reproduced with permission).

given here does not apply to the deep photospheric layers close to the Schwarzschild stability boundary. As Fig. 2 shows, one finds a heating in 3D in comparison to the $1 \mathrm{D}$ structure. The reason is that here an adiabatic stratification is hotter than a structure in radiative equilibrium - given the constraint that all structures must exhibit $T=T_{\text {eff }}$ at $\tau \approx \frac{2}{3}$.

Finally we want to point out that 3D models predict substantial temperature fluctuations in the Schwarzschild-stable layers irrespective of metallicity. Figure 3 (left panel) shows a example of a metal-poor F-dwarf model. The highest plotted atmospheric layers feature temperature fluctuations of over $4000 \mathrm{~K}$ peak-to-peak! While the given example is on the extreme side, substantial fluctuations are nevertheless common.

\section{3D model atmosphere grids}

Large spectroscopic surveys like the LAMOST or Gaia-ESO surveys cover basically all kinds of objects across the Herzsprung-Russell diagram (HRD). If one wants to apply $3 \mathrm{D}$ models to aid the interpretation of survey data one is in need of model grids. Here we want to give an account of the still small number of existing grids - in the author's counting $2 \frac{1}{2}$. As a minimum requirement for a grid we demanded several ten models which should cover the cool part of the main-sequence and the red giant branch.

The first systematic attempt to cover the HRD with 3D models including the metallicity was initiated during the CIFIST (Cosmological Impact of the FIrst STars) project. 


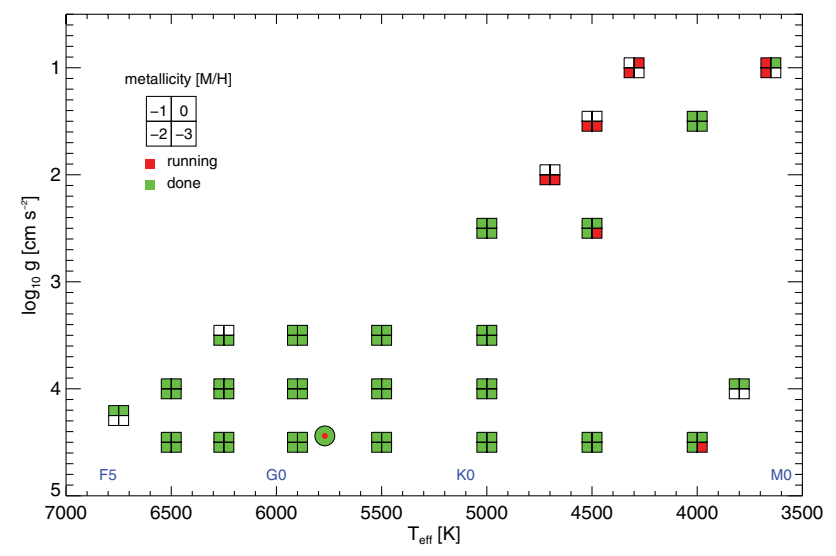

Figure 4. The CIFIST 3D grid as of 2009 containing 77 models. Each $T_{\text {eff }}-\log g$ pair is sub-divided into for metallicities as given in the legend. (from Ludwig et al. 2009; (C)SAIt, reproduced with permission)

Figure 4 shows the status of the CIFIST grid as of 2009. It contained 77 models calculated with the $C^{5}{ }^{5}$ BOLD code (Freytag et al. 2012), covering the main sequence between spectral types F5 and M0 as well as part of the giant branch. Importantly, it contained models between solar and 1/1000 of the solar metallicity. In the meantime the grid was extended, and in particular contains model atmospheres of hydrogen white dwarfs.

Trampedach et al. (2013) constructed a grid of 37 models of solar metallicity with the Nordlund-Stein code (Stein \& Nordlund 1998). This grid we did not count as full since it is restricted to solar metallicity only.

Very recently, Magic et al. (2013) presented an extensive grid of models calculated with the STAGGER code. It contains 220 models. See the contribution of R. Collet in this volume for further discussion.

Hitherto, exploitation of the grids were rather restricted to the study of convection as such. Application to global spectroscopic aspects as desirable for the interpretation of survey data are only at the beginning. The CIFIST grid, as the oldest among the grids, was used in part in two such projects as described later. In any case, the comprehensive application to spectroscopy is a logical step that will happen in the near future.

\section{3D model systematics: getting closer}

With different 3D models around one can address the question what kind of modelling systematics is present, or to which level the various $3 \mathrm{D}$ models agree. Figure 3 (right panel) shows a comparison between the mean temperature structure of solar models calculated with the STAGGER and $\mathrm{CO}^{5}$ BOLD codes (in red). The mean temperatures agree to within $50 \mathrm{~K}$ for $\tau_{500}<1$. This is not perfect, but quite satisfactory considering that discrepancies were reduced by about factor 2 over recent years. The quite satisfactory correspondence also carries over to other properties.

The situation is less favorable for giants. Figure 5 shows that the abundance corrections for molecules in CO5 BOLD models are significantly smaller in magnitude than in Nordlund-Stein/STAGGER models. This is indicative of different temperature structures. The underlying reason is not clear yet but the differences are likely related to different approaches in opacity binning scheme which is used to calculate band-averaged opacities. 


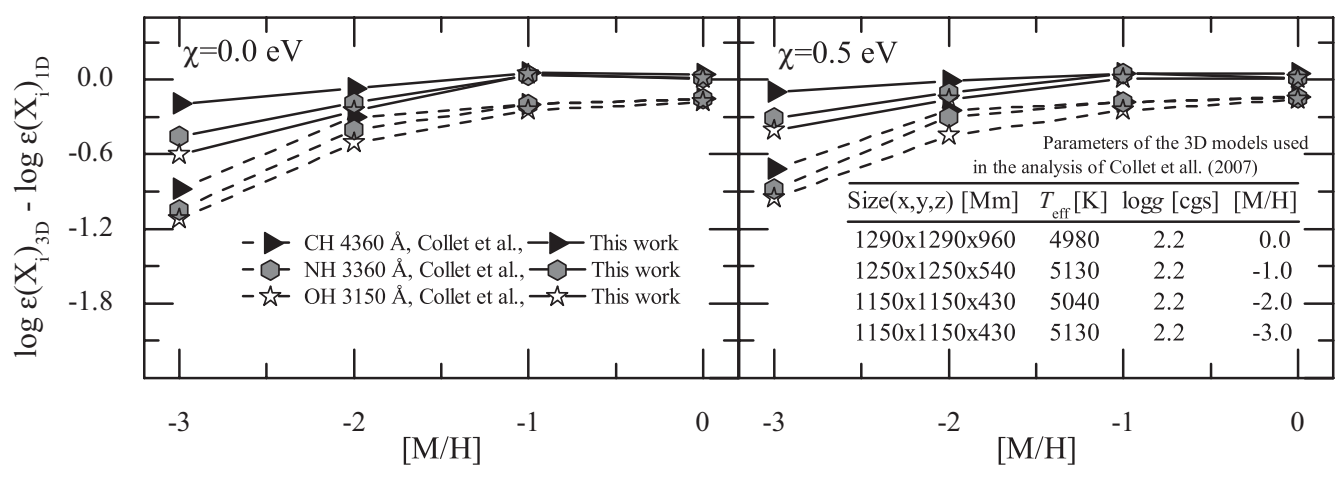

Figure 5. 3D-1D abundance corrections for molecules in giant models of differing metallicity (from Ivanauskas et al. 2010 including data from Collet et al. 2007)

The number of opacity bins - or effective frequency points - used in the radiative transfer is similar in STAGGER and CO ${ }^{5}$ BOLD. This still leaves the possibility that absolute uncertainties in the thermal structures are greater than the comparison between models might indicate. Improving the accuracy of the radiative energy transfer is likely the most crucial aspect for further improving the quality of $3 \mathrm{D}$ models in the future.

\section{Application 1: Microturbulence}

Let us motivate the topic by a quote from a 25 years old paper of two eminent spectroscopists (Griffin \& Holweger, 1989) entitled "An abundance analysis of the Hyades giant $\gamma$ Tauri: an exercise in caution". The paper features a careful consideration of error sources, and the part on microturbulence starts with the statement: "This [the microturbulence] seemingly innocent but potent parameter deserves more respect than is usually gets, as its abuse can produce drastic effects on abundance results". In the following we want to pay some respect from the viewpoint of what $3 \mathrm{D}$ models can tell us about the microturbulence parameter, $\xi_{\text {micro }}$.

In $1 \mathrm{D}$ abundance analyses $\xi_{\text {micro }}$ is usually considered a "nuisance" parameter, meaning that it is not of actual interest but has to be determined together with the other atmospheric parameters $T_{\text {eff }}, \log g$, and metallicity. The microturbulence influences the strength of strong, saturated lines by lifting the saturation and increasing the total equivalent width. $\xi_{\text {micro }}$ is grossly interpreted as effect of the unknown (in 1D!), small-scale atmospheric velocity field, but also helps to compensate for offsets in the thermal structure between model and real stellar atmosphere. Its velocity distribution is usually modelled by a depth-independent Gaussian of fixed width $\xi_{\text {micro }}$ in analogy to thermal line broadening. It is adjusted so that weak and strong lines provide the same abundance. If the other atmospheric parameters are also determined from spectroscopy (e.g., gravity by the ionization balance), their values will depend on the microturbulence. It is this connection that underlies the importance of the microturbulence parameter.

Spectroscopic surveys are typically conducted at rather low spectral resolution and/or $\mathrm{S} / \mathrm{N}$. In such a situation often only strong, saturated lines are measurable. This precludes a determination of $\xi_{\text {micro }}$, and one is in need of a calibration. As we have seen, 3D models predict the atmospheric velocity field, and can provide some theoretical guidance here. We use this rather careful formulation since exploitation of this feature is not far developed yet. Moreover, the observational picture is somewhat messy, hinting at aspects usually not fully accounted for. This may be uncertainties in the atomic line data, or effects of stellar activity and rotation. 


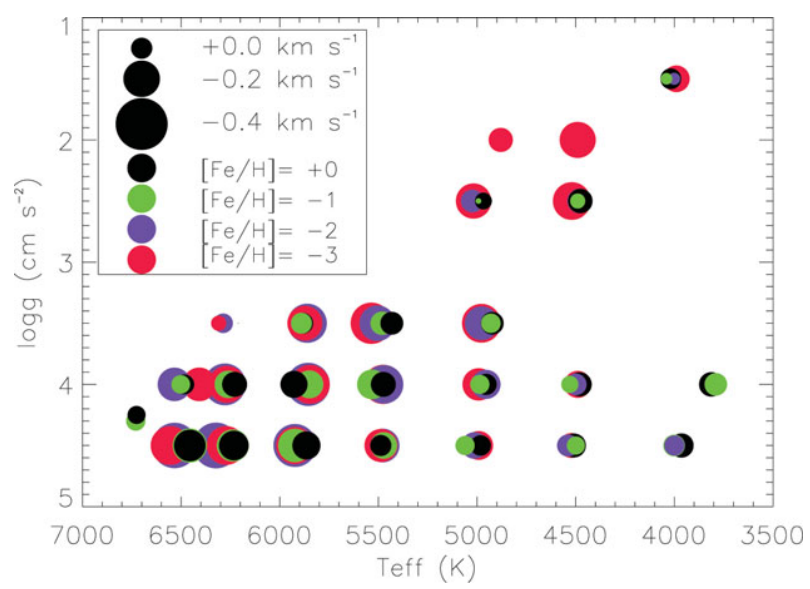

Figure 6. Convective line shifts predicted by 3D models (from Allende Prieto et al. 2013; (C) ESO, reproduced with permission).

An illustrative example of the sometimes perplexing behavior can be found in Table 1 of the paper of Spite et al. (2012). The authors consider a homogeneous sample of metalpoor turn-off stars. For most stars the microturbulence amounts to around $1.5 \mathrm{~km} / \mathrm{s}$. However, for two objects a value of $0.5 \mathrm{~km} / \mathrm{s}$ is derived. While they are among the ones with the highest surface gravity, their exceedingly low value of $\xi_{\text {micro }}$ appears surprising.

At this point we like to digress and discuss a distinct but related application of 3D models: the derivation of line shifts. Figure 6 shows results obtained from models of the CIFIST grid. The wavelength range of about $27 \mathrm{~nm}$ covered by Gaia's Radial Velocity Spectrometer (RVS) was synthesized in 3D taking into account temperature and velocity fluctuations, and cross-correlated with a corresponding synthetic spectrum obtained from a $1 \mathrm{D}$ model. The intention was to obtain the mean line shift in order to be able to correct the measured spectroscopic radial velocity to the actual stellar space motion. On the precision level of the RVS $(\geqslant 1 \mathrm{~km} / \mathrm{s})$ this is a small effect, and is only relevant when averaging over stellar samples. Here, we want to point out that this kind of spectral synthesis calculations are computationally demanding since in $3 \mathrm{D}$ many (several $10^{5}$ ) spectra for all selected spatial-temporal points and ray directions must be calculated and finally averaged. The particular calculations were performed on a machine which at the time ranked among the top ten in the world in terms of its floating point performance.

Coming back to the microturbulence, Fig. 7 shows predicted values of $\xi_{\text {micro }}$ from a small set of $3 \mathrm{D} \mathrm{CO} \mathrm{CO}^{5} \mathrm{BOLD}$ models, here mostly of solar metallicity. $\xi_{\text {micro }}$ was determined by synthesizing iron lines with a range in strength in $3 \mathrm{D}$, and then interpreting their strengths with the help of $1 \mathrm{D}$ models. The 3D lines served as synthetic observation. The 3D predictions deviate noticeably from the calibration derived by Edvardsson et al. (1993), and also show a lower sensitivity to $\log g$. The two points labeled "metal-poor sun" and "Leo star" depict metal-poor models; a metal-poor analog of the Sun at a metallicity of $[\mathrm{M} / \mathrm{H}]=-2$, the other an extremely metal-depleted dwarf at $[\mathrm{M} / \mathrm{H}]=-4$ (discussed in Caffau et al. 2011b). The metal-poor models differ substantially from the ones at solar metallicity. This is not related to an enhanced turbulence in their atmosphere but rather to a mismatch between the $3 \mathrm{D}$ thermal structure and the temperature profile of the $1 \mathrm{D}$ model - as discernible for the metal-poor case in the right panel of Fig. 2.

In Fig. 7 two points are shown for the Sun and Procyon which represent models of different spatial resolution. The derived value of $\xi_{\text {micro }}$ increases for increasing spatial 


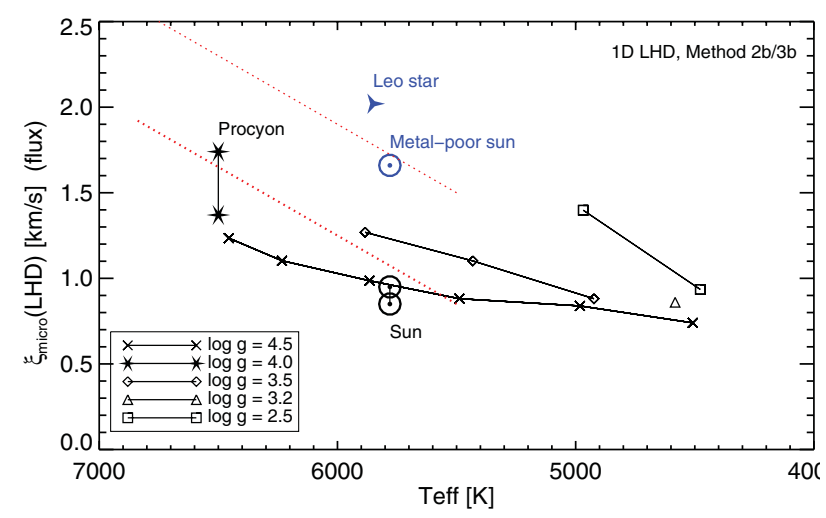

Figure 7. Predicted microturbulence from a small set of 3D models. The red dotted lines depict the calibration of the microturbulence by Edvardsson et al. (1993) for $\log g=4.5$ (lower) and 4.0 (upper line).

resolution. Hence, models of high spatial resolution have to be calculated for a reliable derivation of $\xi_{\text {micro }}$, which is expensive in terms of computing time.

Finally, we want to show an example illustrating the ultimate precision limit to which an abundance analysis can be performed with 1D models. Figure 8 is the diagnostic plot of one of the models depicted in Fig. 7 from which the the microturbulence was determined. It represents an F-dwarf at $T_{\text {eff }}=6300 \mathrm{~K}, \log g=4.5$, and solar metallicity. Its mean temperature structure closely corresponds to the structure of the 1D model used to derive $\xi_{\text {micro }}$. One should recognize that the given situation for determining $\xi_{\text {micro }}$ is next to ideal: the $1 \mathrm{D}$ model has the correct $T_{\text {eff }}$ and $\log g$. All lines are formed in strict LTE - in the "observations" given by the 3D model as well as the $1 \mathrm{D}$ calculations. The atomic line parameters and the line strength are exactly known since the synthetic spectra are noiseless. Following common practice, $\xi_{\text {micro }}$ was determined from Fe I lines with excitation potential $\geqslant 2 \mathrm{eV}$. Low excitation lines of Fe I and Fe II lines are shown for further information. The overall average of the iron abundance of the individual lines comes out close to the abundance put into the 3D synthesis. However, it is remarkable that there remains an overall scatter of $\approx 0.2$ dex peak-to-peak after adjusting $\xi_{\text {micro }}$ to remove the trend of abundance with equivalent width. This is a consequence of the temperature fluctuations which prevail in the atmosphere and whose effect cannot be eliminated by an adjustment of the microturbulence parameter. Note, however, that the abundance scatter could be reduced significantly by adjusting $T_{\text {eff }}$ and $\log g$ of the $1 D$ model such that any trend with excitation potential and any offset between FeI and Fe II lines is minimized. While attractive at first sight this procedure has the drawback to abandon the strict physical meaning of the two parameters $T_{\text {eff }}$ and $\log g$ making them more loosely defined "measures" of the underlying physical quantities.

\section{Application 2: Abundances from molecular lines}

In astronomy, diatomic molecules like $\mathrm{CH}, \mathrm{NH}, \mathrm{OH}, \mathrm{C}_{2}, \mathrm{CN}$, and $\mathrm{CO}$ constitute important abundance indicators. They often exhibit easily detectable broad and strong bands, and at low metallicity their lines are sometimes the only available features. The dissociation equilibria of molecules are often rather T-sensitive. The left panel of Fig. 9 illustrates this for a band of $\mathrm{NH}$ at $336 \mathrm{~nm}$. The contribution function quantifies the differential contribution $d W / d \log (\tau)$ of a particular depth $\tau$ to the total equivalent width $W$. While the depth integral of the $\langle 3 \mathrm{D}\rangle$ and $1 \mathrm{D}$ structure are rather similar, the $3 \mathrm{D}$ model predicts a 


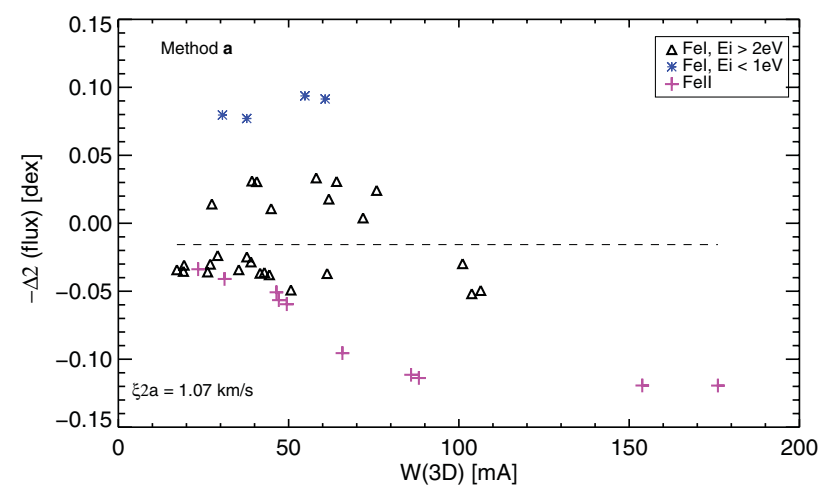

Figure 8. Normalized abundance versus equivalent width $W$ for one model of Fig. 7. $\xi_{\mathrm{micro}}$ was adjusted to remove the trend of abundance with $W$ for Fe I lines with excitation potential $\geqslant 2 \mathrm{eV}$ shown by black triangles. Other symbols see legend.

much larger equivalent width caused by contributions from higher photospheric layers. The difference between $3 \mathrm{D}$ and $\langle 3 \mathrm{D}\rangle$ emphasizes that the temperature fluctuations are decisive.

The right panel of Fig. 9 illustrates another aspect which is often of relevance for the calculation of the number density of a molecular absorber: the formation of a particular molecular is coupled to others via a chemical reaction network. The shown example of the violet $\mathrm{CN}$ band at $388 \mathrm{~nm}$ is an extreme case. The difference between $3 \mathrm{D}$ and $1 \mathrm{D}$ in equivalent width amounts to $\approx 2$ dex. Moreover, the formation of $\mathrm{CN}$ is intimately coupled to CO. In stellar atmospheres $\mathrm{CO}$ is the most stable binary molecule controlling the available amount of carbon. As a consequence, the formation of $\mathrm{CN}$ is extremely sensitive to the carbon-to-oxygen $(\mathrm{C} / \mathrm{O})$ ratio. For $\mathrm{C} / \mathrm{O}>1$ the $\mathrm{CN}$ band becomes very strong. Its formation partially shifts into chromospheric layers so that the model as such and the LTE line formation become rather unreliable. Nevertheless, the effect is clearly present, and we speculate that a similar effect on the strength of the G-band $(\mathrm{CH})$ may have an impact on the derivation of the frequency of carbon-enhanced stars at low metallicity. From the plot it is also apparent that this is a $3 \mathrm{D}$ effect and, again, a consequence of the present temperature fluctuations.

Finally, we point to an application of 3D models to the Galactic evolution of the oxygen abundance. Here, 52 selected 3D models from the CIFIST grid were used to correct 1D oxygen and iron abundances of metal-poor dwarfs. The oxygen abundance was derived from UV-lines of $\mathrm{OH}$, the last remaining abundance indicator of oxygen in extremely metal-poor dwarfs. Figure 10 shows that the trend of the oxygen abundance with metallicity becomes much flatter in $3 \mathrm{D}$ since at lowest metallicities the O-abundances were revised downward by up to a factor of 10 . This corresponds more closely to the results obtained with atomic lines of oxygen in giants, Unfortunately, the departures from LTE of Fe and molecules are largely unexplored in 3D. The "NLTE" labels in the figure refer to corrections obtained from 1D models which give only a rough estimate of what to expect in $3 \mathrm{D}$. This leaves us with significant degree of uncertainty here (for further discussion see the contribution of L. Mashonkina in this volume ).

\section{Take away}

We hope to have shown that 3D model atmospheres constitute the natural path to establish high-fidelity abundances for cool stars. On the to-do list is the exploitation of 

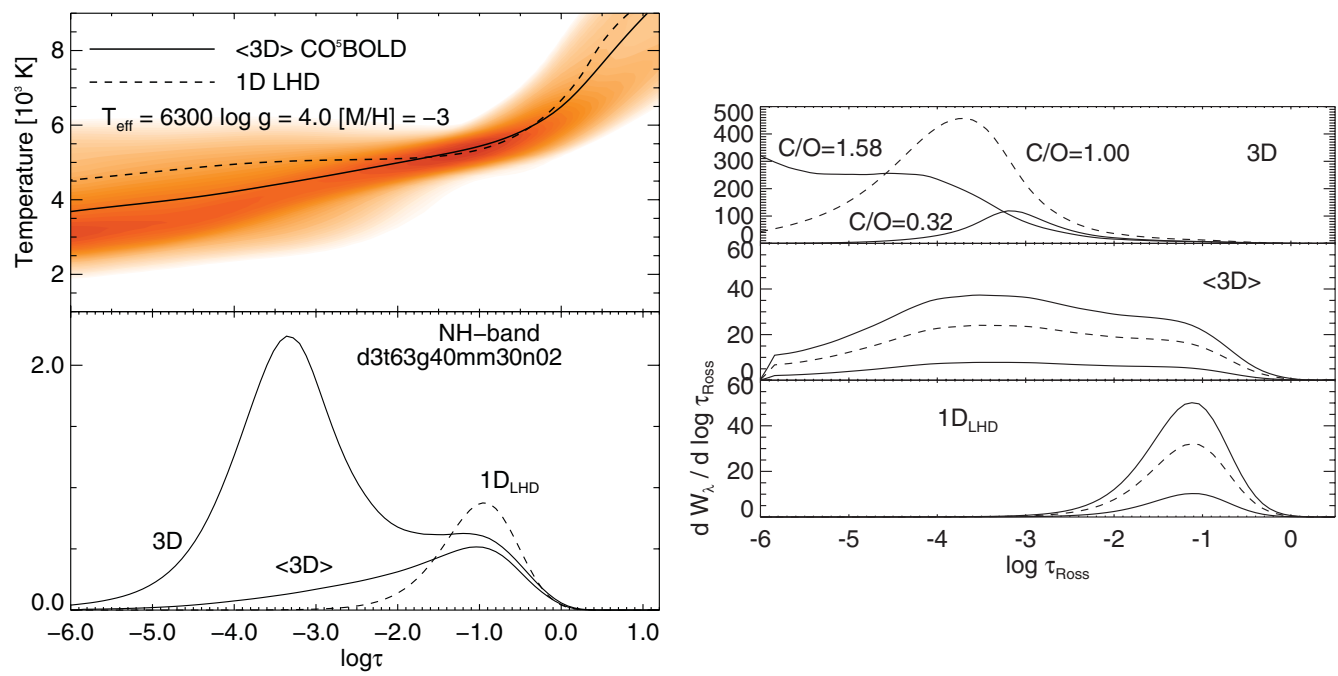

Figure 9. Left: temperature distribution (top) and contribution function (bottom) to the equivalent width of the NH $336 \mathrm{~nm}$ band. Right: Contribution function to the equivalent width of the CN $388 \mathrm{~nm}$ band for different carbon-to-oxygen ratios. (all figures from Bonifacio et al. 2013; (C)SAIt, reproduced with permission)

$1 \mathrm{D}$

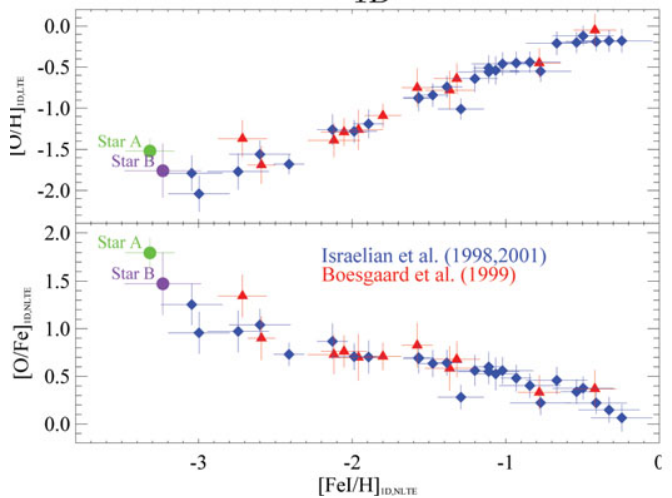

3D-corrected

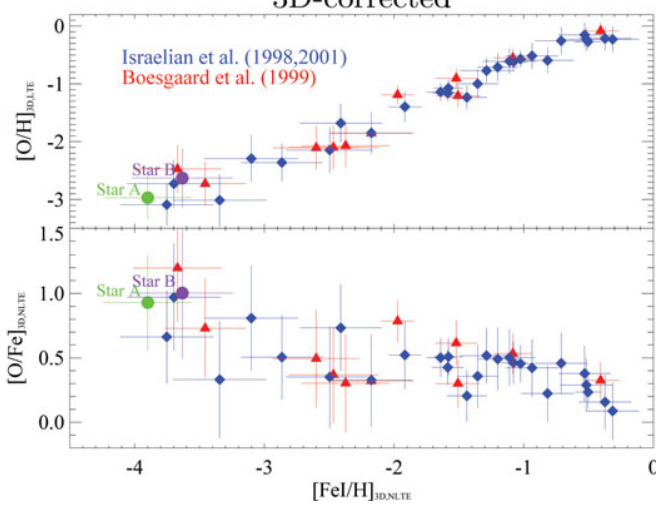

Figure 10. Oxygen abundance and oxygen-to-iron ratio as function of metallicity for metal-poor dwarfs. (from González Hernández et al. 2010; (CESO, reproduced with permission)

existing model grids to support abundance work on large stellar samples as provided by the LAMOST and Gaia-ESO surveys. Model developers still need to work to mitigate existing limitations. On the model side as such improvements of the radiative transfer and spatial resolution are desirable. For spectral synthesis calculations based on 3D models the inclusion of departures from LTE would significantly improve the fidelity of derived abundances. While this is in part a computational challenge, it is also challenging in terms of human resources.

\section{Acknowledgments}

HGL would like to thank the IAU for supporting his participation by a travel grant. EC and HGL acknowledge financial support by the Sonderforschungsbereich SFB 881 "The Milky Way System" (subproject A4) of the German Research Foundation (DFG). 
B.F. acknowledges financial support from the Agence Nationale de la Recherche (ANR), and the "Programme Nationale de Physique Stellaire" (PNPS) of CNRS/INSU, France.

\section{References}

Allende Prieto, C., Koesterke, L., Ludwig, H.-G., Freytag, B., \& Caffau, E., 2013, A $\&$ A, 550, A103

Asplund, M., Grevesse, N., Sauval, A. J., Allende Prieto, C., \& Kiselman, D., 2004, A\& A, 417, 751

Asplund, M., Lambert, D. L., Nissen, P. E., Primas, F., \& Smith, V. V., 2006, ApJ, 644, 229

Asplund, M., Grevesse, N., Sauval, A. J., \& Scott, P., 2009, ARAA, 47, 481

Beeck, B., Collet, R., Steffen, M., Asplund, M., Cameron, R. H., Freytag, B., Hayek, W., Ludwig, H.-G., \& Schüssler, M., 2012, A\& A, 539, A121

Bonifacio, P., Caffau, E., Ludwig, H.-G., Spite, M., Plez, B., Steffen, M., \& Spite, F., 2013, MemSAIt, in press

Caffau, E., Ludwig, H.-G., Steffen, M., Ayres, T. R., Bonifacio, P., Cayrel, R., Freytag, B., \& Plez, B, 2008, AשAA, 488, 1031

Caffau, E., Ludwig, H.-G., Steffen, M., Freytag, B., \& Bonifacio, P., 2011a, Solar Phys., 268, 255

Caffau, E., Bonifacio, P., François, P., Sbordone, L., Monaco, L., Spite, M. Spite, F., Ludwig, H.-G., Cayrel, R., Zaggia, S., Hammer, F., Randich, S., Molaro, P., \& Hill, V., 2011b, Nature, 477,67

Cayrel, R., Steffen, M., Chand, H., Bonifacio, P., Spite, M., Spite, F., Petitjean, P., Ludwig, H.-G., \& Caffau, E., 2007, A\&广A, 473, L37

Collet, R., Asplund, M., \& Trampedach, R., 2007, A\&A, 469, 687

Edvardsson, B., Andersen, J., Gustafsson, B., Lambert, D. L., Nissen, P. E., \& Tomkin, J., 1993, $A \mathscr{E} A, 275,101$

Freytag, B., Steffen, M., Ludwig, H.-G., Wedemeyer-Böhm, S., Schaffenberger, W., \& Steiner, O., 2012, J. Comp. Phys., 231, 919

González Hernández, J. I., Bonifacio, P., Ludwig, H.-G., Caffau, E., Behara, N. T., \& Freytag, B., 2010, $A \& A, 519, \mathrm{~A} 46$

Griffin, R. E. M, \& Holweger, H., 1988, AधA, 214, 249

Magic, Z., Collet, R., Asplund, M., Trampedach, R., Hayek, W., Chiavassa, A., Stein, R. F., \& Nordlund, A., 2013, $A \mathscr{E} A$, in press

Ivanauskas, A., Kucinskas, A., Ludwig, H.-G., \& Caffau, E., 2010, in: Proceedings of the 11th Symposium on Nuclei in the Cosmos, published online

Lind, K., Melendez, J., Asplund, M., Collet, R., \& Magic, Z., 2013, A\&AA, 554, A96

Ludwig, H.-G., Caffau, E., Steffen, M., Freytag, B., Bonifacio, P., \& Kučinskas, A., 2009, MemSAIt, 80, 711

Spite, M., Andrievsky, S. M., Spite, F., Caffau, E., Korotin, S. A., Bonifacio, P., Ludwig, H.-G., François, P., \& Cayrel, R., 2012, A\&A, 541, A143

Steffen, M., Cayrel, R., Caffau, E., Bonifacio, P., Ludwig, H.-G., \& Spite, M., 2012, MemSAIt, 22,152

Stein, R. F., \& Nordlund, A., 1998, ApJ, 499, 914

Trampedach, R., Asplund, M., Collet, R., Nordlund, Å., \& Stein, R. F., 2013, ApJ, 769, 18

\section{Discussion}

Jorge MelÉndez: 1) Are the most important discrepancies with 1D in the outermost layers? 2) The NLTE effects may be more important for those layers, so that NLTE+3D may be closer to the $1 \mathrm{D}$ models, right?

HANS-Günther Ludwig: 1) Commonly, differences in the mean structure and the level of fluctuations become greater towards the outermost layers. For spectroscopy, it depends on the actual line formation properties whether the outermost or deeper layers are more important. 2) Indeed, NLTE effects grow towards the outermost layers. Experience (from 
detailed calculations) so far shows that 3D abundances get closer to 1D if NLTE effects are properly accounted for. However, this needs to be worked out on a case-by-case basis. Moreover, I conjecture that NLTE effects for molecular features will be rather modest.

JAMES Binney: My naive understanding of the properties of $3 \mathrm{D}$ models is that the spectrum is formed in regions of different temperatures, densities, etc. Could one diminish the computational load by simply introducing a degree of randomness into the surface properties without integrating the hydro equations over a long time?

HANS-GÜNTHER LUDWIG: We already worked on a naive approach by trying to classify the atmospheric structure into components with common properties (mostly a similar vertical temperature profile), and restricting the spectral synthesis to these few components. The outcome was not satisfactory so far. A more involved statistical approach (as you point to) is perhaps necessary. If successful it would be of great practical importance.

JohAnnes Andersen: Would you comment on the abundance effects of the enhanced convective penetration in $3 \mathrm{D}$ ?

HANS-GüNTHER LuDWIG: Besides the effects on the thermal structure and velocity field discussed in this paper, mixing and the formation of shocks are further aspects that can play a role. In particular, the cloud formation in very cool atmospheres (e.g., in brown dwarfs) is linked to convective penetration. The formation of shocks can lead to substantial deviations from LTE, however, it is usually confined to chromospheric layers and, hence, has limited impact on typical abundance work. 